\title{
Theory of doorway states for one-nucleon transfer reactions. II. Model-independent study of nuclear correlation effects
}

\author{
B.L. Birbrair \& V.I. Ryazanov \\ PNPI, Gatchina 188350, Russia \\ birbrair@thd.pnpi.spb.ru
}

\begin{abstract}
The correlation effects in nuclei owing to which the nuclear wave functions are different from the Slater determinants are studied on the basis of the theory of Ref.[1]. The calculated numbers of nucleons out of the nuclear Fermi-surface are in reasonable agreement with the finding from the high-momentum components of the nucleon momentum distributions in nuclei. The problems concerning the nuclear binding energy are also discussed.
\end{abstract}

\section{Introduction}

The nucleon-nucleon interaction still remains one of the central problems of the nuclear structure theory. It seems natural to use the free-space forces, but the most of the nuclear structure theorists prefer the effective ones instead. The basic motivations are as follows.

(a) Historically the first one arose from the wide-spread belief in sixties that the free-space $N N$ potential has a hard repulsive core. Clearly such interaction does not apply directly because of the divergence of finite-order Feynman diagrams. The most popular way to overcome this difficulty is to calculate the Brueckner $G$-matrix and use it as the effective interaction in the Hartree-Fock problem. This is the Hartree-Fock-Brueckner approximation, see [2] and the references therein for details. But soon it became clear that the description of the two-nucleon system, i.e. the deuteron properties and the elastic $N N$ scattering phase shifts below the pion production threshold, does not require the hard repulsive core. As a result all the contemporary $N N$ potentials are of soft core character, and so the above difficulty does not really exist. In such conditions the calculation of the Brueckner $G$-matrix is not compulsory.

(b) The renormalization of the free-space interaction due to the medium polarization effects. But such effects are treated by conventional methods of quantum many-body theory, and therefore the above reason is not the argument for the effective forces.

(c) The medium QCD-renormalization due to the fact that the quark composition of the QCD vacuum is changed in nuclear medium thus leading to the changes of both the mesons 
and the meson-nucleon vertices [3]-6]. Such processes can hardly be described in all details since the exact theory does not yet exist for the nonperturbative region. Nevertheless, at least one exact statement can be done. The QCD-renormalized interaction is the functional of nuclear density possessing the following obvious property: it turns into the free-space one in the zero-density limit. For this reason it can be represented as the following functional expansion:

$$
\begin{aligned}
f_{Q C D-r}\left(\mathbf{r}_{1} \mathbf{r}_{2}\{\rho\}\right) & =f_{2}\left(\left|\mathbf{r}_{1}-\mathbf{r}_{2}\right|\right)+\int f_{3}\left(\left|\mathbf{r}_{1}-\mathbf{r}_{2}\right|,\left|\mathbf{r}_{1}-\mathbf{r}^{\prime}\right|\right) \rho\left(r^{\prime}\right) d^{3} \mathbf{r}^{\prime}+ \\
& +\iint f_{4}\left(\left|\mathbf{r}_{1}-\mathbf{r}_{2}\right|,\left|\mathbf{r}_{1}-\mathbf{r}^{\prime}\right|,\left|\mathbf{r}_{1}-\mathbf{r}^{\prime \prime}\right|\right) \rho\left(r^{\prime}\right) \rho\left(r^{\prime \prime}\right) d^{3} \mathbf{r}^{\prime} d^{3} \mathbf{r}^{\prime \prime}+\cdots
\end{aligned}
$$

In this way we conclude that the medium QCD-renormalization is equivalent to the existence of many-particle $N N$ forces in addition to the two-particle ones. This conclusion is confirmed by the fact that the physics of strong interaction is essentially nonlinear. But the nonlinearity automatically leads to many-particle forces.

The above reasons are grounds for our starting point: both the two-particle and manyparticle $N N$ forces must be taken into account for the treatment of nuclear structure.

The three-particle $N N$ forces are indeed included for the calculations of few-nucleon systems [7]. But the following questions arises: is this sufficient for complex nuclei ? This point as well as a number of those concerning the nuclear structure might be elucidated if the modelindependent object would exist in nuclei.

Our approach is based on the fact that such objects do really exist. As discussed in our previous work [1] they are the doorway states for one-nucleon transfer reaction. We showed that

(i) at least the three-particle repulsion and the four-particle attraction must be taken into account in addition to the two-particle forces;

(ii) the nuclear relativity is really existing phenomenon rather than the hypothesis of J.D. Walecka [8];

(iii) the dominant contribution to the isovector nuclear potential is provided by the manyparticle forces;

(iv) the observed spectra of the doorway states can be used to specify the neutron density distributions in nuclei; such specified densities are indeed obtained for the closed-shell nuclei ${ }^{40} \mathrm{Ca},{ }^{90} \mathrm{Zr}$ and ${ }^{208} \mathrm{~Pb}$.

In the present work our approach is applied to the "empirical" studies of nuclear correlation effects owing to which the nuclear wave functions are different from the Slater determinants. Such effects are treated by a variety of approximate methods since the exact ones do not exist. For this reason it is very important to get a model-independent quantitative information about the above effects. The possibility provided by our approach is based on the fact that the single-particle states of nucleon in nuclear static field (which are just the doorway states for one-nucleon transfer reactions [1]) are correlation-free objects in contrast to the LandauMigdal quasiparticles [9] (which include the correlations by definition) and single-particle states in nuclear Hartree-Fock calculations with effective forces (where the correlations are implicitly included in the phenomenological effective force parameters). So, calculating the correlationfree quantities and comparing them with the observed ones we get a quantitative measure of 
the correlation effects. In Sect.2 this procedure is applied to the nucleon density distributions whereas the problems concerning the nuclear binding energy are discussed in Sect.3.

\section{Density distributions}

The nucleon density distribution in the ground state of nucleus $A$ is (see [1] for the notations)

$$
\begin{aligned}
\rho(r) & =\left\langle A_{0}\left|\psi^{+}(x) \psi(x)\right| A_{0}\right\rangle=\sum_{j}^{(A-1)}\left\langle A_{0}\left|\psi^{+}(x)\right|(A-1)_{j}\right\rangle\left\langle(A-1)_{j}|\psi(x)| A_{0}\right\rangle= \\
& =\sum_{j}^{(A-1)} \Psi_{j}^{+}(x) \Psi_{j}(x)=\int_{C} \frac{d \varepsilon}{2 \pi i} G(x, x ; \varepsilon) .
\end{aligned}
$$

The integration contour $C$ includes the real axis and the infinite radius semicircle in the upper part of the complex $\varepsilon$ plane. As seen from Eq.(1) the density is expressed through the singleparticle amplitudes of the $(A-1)$ nuclear states. Expanding them over the complete set of the doorway states,

$$
\Psi_{j}(x)=\sum_{\lambda} C_{j}^{(\lambda)} \psi_{\lambda}(x)
$$

and putting Eq.(3) into Eq.(2) we get

$$
\rho(r)=\sum_{\lambda} n_{\lambda}\left|\psi_{\lambda}(x)\right|^{2}+2 \sum_{\substack{\lambda \nu \\ \nu>\lambda}} \rho_{\lambda \nu} \psi_{\lambda}^{+}(x) \psi_{\nu}(x)
$$

where

$$
n_{\lambda}=\rho_{\lambda \lambda}=\sum_{j}^{(A-1)}\left|C_{j}^{(\lambda)}\right|^{2}=\sum_{j}^{(A-1)} s_{j}^{(\lambda)}, \quad \rho_{\lambda \nu}=\sum_{j}^{(A-1)} C_{j}^{(\lambda)^{*}} C_{j}^{(\nu)}
$$

(actually the coefficients $C_{j}^{(\lambda)}$ are real quantities if the parity violation due to weak interaction is disregarded). The diagonal elements $\rho_{\lambda \lambda}=n_{\lambda}$ are the doorway state occupation numbers. Indeed, the particle number is

$$
N=\int \rho(r) d^{3} \mathbf{r}=\sum_{\lambda} n_{\lambda}
$$

since the nondiagonal elements do not contribute because of the orthogonality between $\psi_{\lambda}$ and $\psi_{\nu}$. The quantities $n_{\lambda}$ and $\rho_{\lambda \nu}$ obey the following limitations:

$$
0<n_{\lambda}<1, \quad\left|\rho_{\lambda \nu}\right|<\frac{1}{2}\left(n_{\lambda}+n_{\nu}\right) .
$$

The first follows from the fact that the doorway states are distributed over the actual ones of both the $(A-1)$ and $(A+1)$ nuclei, see Eq.(13c) in Ref. [1], whereas the second is a consequence of the Cauchy-Bunjakowsky inequality.

The facts $n_{\lambda}<1$ and $\rho_{\lambda \nu} \neq 0$ reflect the Fermi surface smearing due to the correlation effects. The quantities $n_{\lambda}$ and $\rho_{\lambda \nu}$ carrying the quantitative information about these effects 


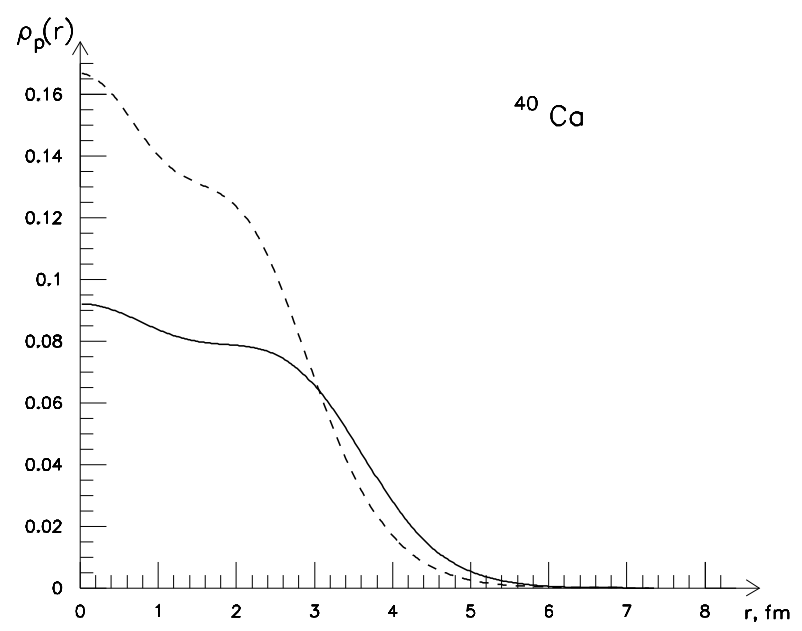

Figure 1: The observed (full line) and correlation-free (dashed one) proton density distributions in ${ }^{40} \mathrm{Ca}$.

can be found "empirically" by considering the relation (4) together with the limitations (7) as equations for $n_{\lambda}$ and $\rho_{\lambda \nu}$. The results will be published elsewhere.

As a result of the correlations a part of nucleons is out of the nuclear Fermi-surface. The number $N_{\text {out }}$ of such nucleons is calculated by comparing the observed density distribution with the correlation-free one

$$
\rho_{c f}(r)=\sum_{\lambda} \Theta\left(\varepsilon_{F}-\varepsilon_{\lambda}\right)\left|\psi_{\lambda}(x)\right|^{2} .
$$

$\varepsilon_{F}$ is the Fermi level energy. Such comparison is shown in Fig.1 for the proton density distribution in ${ }^{40} \mathrm{Ca}$. As seen from the figure the correlation-free density contains more nucleons in the inner region $0<r<r_{i}$ than the observed one, $r_{i}$ is the intersection point. The situation in the outer region $r_{i}<r<\infty$ is clearly opposite since both densities correspond to the same number of nucleons. The number of redistributed nucleons is

$$
N_{\text {out }}=4 \pi \int_{0}^{r_{i}}\left[\rho_{c f}(r)-\rho(r)\right] r^{2} d r=4 \pi \int_{r_{i}}^{\infty}\left[\rho(r)-\rho_{c f}(\rho)\right] r^{2} d r .
$$

$r_{i}$ is the intersection point: $\rho_{c f}\left(r_{i}\right)=\rho\left(r_{i}\right)$. This is just the $N_{\text {out }}$ because the only reason for the redistribution is the Fermi-surface smearing due to the correlations.

The $N_{\text {out }}$ numbers in doubly closed-shell nuclei are shown in Table 1 . As seen from the table these nucleons are a rather appreciable part of the total mass number. To our knowledge this fact was first mentioned by Frankfurt and Strikman [10 on the basis of the analysis of high-momentum components (i.e. those with $k>300 \mathrm{MeV} / \mathrm{c}$ ) of the nucleon momentum distributions in nuclei. According to their most recent results for these data [1] the $A_{\text {out }} / A$ ratio is $(20 \pm 3) \%$ for heavy nuclei. This is in reasonable agreement with our results.

It is worth mentioning that our calculations do not tell anything about the nature of the underlying correlations whereas the high-momentum tales of the momentum distributions arise 
Table 1: $N_{\text {out }}$ numbers in doubly closed-shell nuclei

\begin{tabular}{ccccc}
\hline & ${ }^{16} \mathrm{O}$ & ${ }^{40} \mathrm{Ca}$ & ${ }^{90} \mathrm{Zr}$ & ${ }^{208} \mathrm{~Pb}$ \\
& & & & \\
$N_{\text {out }}$ & 1.10 & 3.15 & 7.34 & 13.15 \\
$Z_{\text {out }}$ & 1.15 & 3.53 & 6.46 & 13.22 \\
$A_{\text {out }}$ & 2.25 & 6.68 & 13.80 & 26.37 \\
$A_{\text {out }} / A, \%$ & 14 & 16.7 & 15.3 & 12.7 \\
\hline
\end{tabular}

from the $N N$ interactions at short distances [10, 11]. Therefore the reasonable agreement between the two results gives rise to the conclusion that the main reason for the Fermi-surface smearing in doubly closed-shell nuclei is due to the short-range correlations.

\section{$3 \quad$ Binding energy}

We also can calculate the part of the nuclear binding energy which is caused by the motion of nucleons in nuclear static field. Such static energy partly includes the correlation effects since it is expressed through the observed nucleon density distributions. The comparison between the observed binding energy $\mathcal{E}_{b}$ and the static one $\mathcal{E}_{s t}$ gives the measure of the proper correlation energy of nucleus.

To make this point clear let us derive the exact expression for the binding energy. Following the procedure of Ref.[9] we get

$$
\begin{aligned}
& \left.\mathcal{E}_{b}=\int d x \int_{c} \frac{d \varepsilon}{2 \pi i} \operatorname{tr} \hat{k}_{x} G(x, x ; \varepsilon)+\frac{1}{2} \iint d x d x_{1} \iint_{c} \frac{d \varepsilon d \varepsilon_{1}}{(2 \pi i)^{2}} f_{2}\left(\left|\mathbf{r}-\mathbf{r}_{1}\right|\right) ; \omega\right) K_{2}\left(x, x_{1} ; x, x_{1} ; \varepsilon, \varepsilon_{1}\right)+ \\
& +\quad \frac{1}{3} \iiint_{1} d x d x_{1} d x_{2} \iiint_{c} \frac{d \varepsilon d \varepsilon_{1} d \varepsilon_{2}}{(2 \pi i)^{3}} f_{3}\left(\left|\mathbf{r}-\mathbf{r}_{1}\right|,\left|\mathbf{r}-\mathbf{r}_{2}\right| ; \omega, \omega_{1}\right) K_{3}\left(x, x_{1}, x_{2} ; x, x_{1}, x_{2} ; \varepsilon, \varepsilon_{1}, \varepsilon_{2}\right)+ \\
& +\quad \frac{1}{4} \iiint \int d x d x_{1} d x_{2} d x_{3} \iiint \int_{c} \frac{d \varepsilon d \varepsilon_{1} d \varepsilon_{2} d \varepsilon_{3}}{(2 \pi i)^{4}} f_{4}\left(\left|\mathbf{r}-\mathbf{r}_{1}\right|,\left|\mathbf{r}-\mathbf{r}_{2}\right|,\left|\mathbf{r}-\mathbf{r}_{3}\right| ; \omega, \omega_{1}, \omega_{2}\right) \times \\
& \times \quad K_{4}\left(x, x_{1}, x_{2}, x_{3} ; x, x_{1}, x_{2}, x_{3} ; \varepsilon, \varepsilon_{1}, \varepsilon_{2}, \varepsilon_{3}\right)
\end{aligned}
$$

where $K\left(x_{1}, \ldots x_{n} ; x_{1}^{\prime} \ldots x_{n}^{\prime} ; \varepsilon_{1} \ldots \varepsilon_{n}\right)$ are the $n$-particle Green functions. We accounted for the three- and four-particle forces in addition to the two-particle ones as well as the possible dependence of the interactions on the appropriate energy transfers $\omega_{i}$. In these terms the single-particle Green function is

$$
\begin{aligned}
& \left(\varepsilon-\hat{k}_{x}\right) G\left(x, x^{\prime} ; \varepsilon\right)=\delta\left(x-x^{\prime}\right)+\int d x_{1} \int_{c} \frac{d \varepsilon_{1}}{2 \pi i} f_{2}\left(\left|\mathbf{r}-\mathbf{r}_{1}\right| ; \omega\right) K_{2}\left(x, x_{1} ; x^{\prime}, x_{1}^{\prime} ; \varepsilon, \varepsilon_{1}\right)+ \\
& +\quad \iint d x_{1} d x_{2} \iint_{c} f_{3}\left(\left|\mathbf{r}-\mathbf{r}_{1}\right|,\left|\mathbf{r}-\mathbf{r}_{2}\right| ; \omega, \omega_{1}\right) K_{3}\left(x, x_{1}, x_{2} ; x^{\prime}, x_{1}, x_{2} ; \varepsilon, \varepsilon_{2}\right)+
\end{aligned}
$$




$$
\begin{aligned}
& +\quad \iiint d x_{1} d x_{2} d x_{3} \iiint_{c} \frac{d \varepsilon_{1} d \varepsilon_{2} d \varepsilon_{3}}{(2 \pi i)^{3}} f_{4}\left(\left|\mathbf{r}-\mathbf{r}_{1}\right|,\left|\mathbf{r}-\mathbf{r}_{2}\right|,\left|\mathbf{r}-\mathbf{r}_{3}\right| ; \omega, \omega_{1}, \omega_{2}\right) \times \\
& \times \quad K_{4}\left(x, x_{1}, x_{2}, x_{3} ; x^{\prime}, x_{1}, x_{2}, x_{3} ; \varepsilon, \varepsilon_{1}, \varepsilon_{2}, \varepsilon_{3}\right) .
\end{aligned}
$$

On the other hand the Dyson equation is

$$
\left(\varepsilon-\hat{k}_{x}\right) G\left(x, x^{\prime} ; \varepsilon\right)=\delta\left(x-x^{\prime}\right)+\int d x_{1} M\left(x, x_{1} ; \varepsilon\right) G\left(x_{1}, x^{\prime} ; \varepsilon\right),
$$

and therefore

$$
\begin{aligned}
& \left.\int M\left(x, x_{1} ; \varepsilon\right) G\left(x_{1}, x^{\prime} ; \varepsilon\right) d x_{1}=\int d x_{1} \int_{c} \frac{d \varepsilon_{1}}{2 \pi i} f_{2}\left(\mid \mathbf{r}-\mathbf{r}_{1}\right) ; \omega\right) K_{2}\left(x, x_{1} ; x^{\prime}, x_{1} ; \varepsilon, \varepsilon_{1}\right)+ \\
& +\quad \iint d x_{1} d x_{2} \iint_{c} \frac{d \varepsilon_{1} d \varepsilon_{2}}{(2 \pi i)^{2}} f_{3}\left(\left|\mathbf{r}-\mathbf{r}_{1}\right|,\left|\mathbf{r}-\mathbf{r}_{2}\right| ; \omega, \omega_{1}\right) K_{3}\left(x, x_{1}, x_{2} ; x^{\prime}, x_{1}, x_{2} ; \varepsilon, \varepsilon_{1}, \varepsilon_{2}\right)+ \\
& +\quad \iiint d x_{1} d x_{2} d x_{3} \iiint_{c} \frac{d \varepsilon_{1} d \varepsilon_{2} d \varepsilon_{3}}{(2 \pi i)^{3}} f_{4}\left(\left|\mathbf{r}-\mathbf{r}_{1}\right|,\left|\mathbf{r}-\mathbf{r}_{2}\right|,\left|\mathbf{r}-\mathbf{r}_{3}\right| ; \omega, \omega_{1}, \omega_{2}\right) \times \\
& \quad \times \quad K_{4}\left(x, x_{1}, x_{2}, x_{3} ; x^{\prime}, x_{1}, x_{2}, x_{3} ; \varepsilon, \varepsilon_{1}, \varepsilon_{2}, \varepsilon_{3}\right) .
\end{aligned}
$$

As seen from Eqs. (10) and (13) the binding energy can be written as

$$
\begin{aligned}
& \mathcal{E}_{b}=\iint d x d x^{\prime} \int_{c} \frac{d \varepsilon}{2 \pi i}\left(t r \hat{k}_{x} \delta\left(x-x^{\prime}\right)+M\left(x, x^{\prime} ; \varepsilon\right)\right) G\left(x^{\prime}, x ; \varepsilon\right)- \\
& -\quad \frac{1}{2} \iint d x d x_{1} \iint_{c} \frac{d \varepsilon d \varepsilon_{1}}{(2 \pi i)^{2}} f_{2}\left(\left|\mathbf{r}-\mathbf{r}_{1}\right| ; \omega\right) K_{2}\left(x, x_{1} ; x, x_{1} ; \varepsilon, \varepsilon_{1}\right)- \\
& -\quad \frac{2}{3} \iiint d x d x_{1} d x_{2} \iiint_{c} \frac{d \varepsilon d \varepsilon_{1} d \varepsilon_{2}}{(2 \pi i)^{3}} f_{3}\left(\left|\mathbf{r}-\mathbf{r}_{1}\right|,\left|\mathbf{r}-\mathbf{r}_{2}\right| ; \omega, \omega_{1}\right) K_{3}\left(x, x_{1}, x_{2} ; x, x_{1}, x_{2} ; \varepsilon_{1}, \varepsilon_{2}\right)- \\
& -\quad \frac{3}{4} \iiint \int d x d x_{1} d x_{2} d x_{3} \iiint \int_{c} \frac{d \varepsilon d \varepsilon_{1} d \varepsilon_{2} d \varepsilon_{3}}{(2 \pi i)^{4}} f_{4}\left(\left|\mathbf{r}-\mathbf{r}_{1}\right|,\left|\mathbf{r}-\mathbf{r}_{2}\right|,\left|\mathbf{r}-\mathbf{r}_{3}\right|, \omega, \omega_{1}, \omega_{2}\right) \times \\
& \times \quad K_{4}\left(x, x_{1}, x_{2}, x_{3} ; x, x_{1}, x_{2}, x_{3} ; \varepsilon, \varepsilon_{1}, \varepsilon_{2}, \varepsilon_{3}\right) .
\end{aligned}
$$

Taking into account the spectral representation of $G\left(x, x^{\prime} ; \varepsilon\right)$, see Eq.(5) in Ref. [1], and performing the integration over $\varepsilon$ we get the following expression for the first term in the rhs:

$$
\mathcal{E}_{b}^{(1)}=\sum_{j}^{(A-1)} \int d x \Psi_{j}^{+}(x)\left(\hat{k}_{x} \Psi_{j}(x)+\int M\left(x, x^{\prime} ; E_{j}\right) \Psi_{j}\left(x^{\prime}\right) d x^{\prime}\right) .
$$

As follows from the Dyson equation (12) and the above spectral representation the amplitudes $\Psi_{j}(x)$ obey the equation

$$
\hat{k}_{x} \Psi_{j}(x)+\int M\left(x, x^{\prime} ; E_{j}\right) \Psi_{j}\left(x^{\prime}\right) d x^{\prime}=E_{j} \Psi_{j}(x)
$$

and therefore

$$
\mathcal{E}_{b}^{(1)}=\sum_{j}^{(A-1)} E_{j} \int\left|\Psi_{j}(x)\right|^{2} d x=\sum_{\lambda} \sum_{j}^{(A-1)} E_{j} s_{j}^{(\lambda)}
$$


(we accounted for the expansion (3)).

In general case the many-particle Green functions $K_{n}\left(x_{1}, \ldots x_{n} ; x_{1}^{\prime}, \ldots x_{n}^{\prime} ; \varepsilon_{1}, \ldots \varepsilon_{n}\right)$ obey the infinite system of integro-differential equations the Eq.(11) being the first one. As mentioned above the exact methods of solution do not exist. It should be also mentioned that the approximate methods, see [12] and the references therein, are developed for the instantaneous two-particle forces only. For these reasons the exact calculation of nuclear binding energy is impossible at present. Therefore the calculation of the static energy is of importance.

Both the static nuclear field and the static energy are obtained from the above relations by putting

$$
K_{n}\left(x_{1}, \ldots x_{n} ; x_{1}^{\prime}, \ldots x_{n}^{\prime} ; \varepsilon_{1}, \ldots \varepsilon_{n}\right)=\prod_{i=1}^{n} G\left(x_{i}, x_{i}^{\prime} ; \varepsilon_{i}\right),
$$

i.e. neglecting the difference between the many-particle Green functions and the unsymmetrized products of the single-particle ones. This difference arises from both the antisymmetrization and the higher-order terms of the perturbation theory, i.e. just the effects leading to the proper correlation energy. Putting Eq.(18) into Eqs.(13) and (14) we get (all the energy transfers $\omega_{i}$ vanish in this case)

$$
\begin{aligned}
& U_{s t}(r)=\int f_{2}\left(\left|\mathbf{r}-\mathbf{r}_{1}\right|\right) \rho\left(r_{1}\right) d^{3} \mathbf{r}_{1}+\iint f_{3}\left(\left|\mathbf{r}-\mathbf{r}_{1}\right|,\left|\mathbf{r}-\mathbf{r}_{2}\right|\right) \rho\left(r_{1}\right) \rho\left(r_{2}\right) d^{3} \mathbf{r}_{1} d^{3} \mathbf{r}_{2}+ \\
& +\quad \iiint f_{4}\left(\left|\mathbf{r}-\mathbf{r}_{1}\right|,\left|\mathbf{r}-\mathbf{r}_{2}\right|,\left|\mathbf{r}-\mathbf{r}_{3}\right|\right) \rho\left(r_{1}\right) \rho\left(r_{2}\right) \rho\left(r_{3}\right) d^{3} \mathbf{r}_{1} d^{3} \mathbf{r}_{2} d^{3} \mathbf{r}_{3} ; \\
& \mathcal{E}_{s t}=\sum_{j}^{(A-1)} \int \Psi_{j}^{+}(x)\left(\hat{k}_{x}+U_{s t}(r)\right) \Psi_{j}(x) d x-\frac{1}{2} \iint f_{2}\left(\left|\mathbf{r}-\mathbf{r}_{1}\right|\right) \rho(r) \rho\left(r_{1}\right) d^{3} \mathbf{r} d^{3} \mathbf{r}_{1}- \\
& -\quad \frac{2}{3} \iiint f_{3}\left(\left|\mathbf{r}-\mathbf{r}_{1}\right|,\left|\mathbf{r}-\mathbf{r}_{2}\right|\right) \rho(r) \rho\left(r_{1}\right) \rho\left(r_{2}\right) d^{3} \mathbf{r} d^{3} \mathbf{r}_{1} d^{3} \mathbf{r}_{2}- \\
& -\frac{3}{4} \iiint \int f_{4}\left(\left|\mathbf{r}-\mathbf{r}_{1}\right|,\left|\mathbf{r}-\mathbf{r}_{2}\right|,\left|\mathbf{r}-\mathbf{r}_{3}\right|\right) \rho(r) \rho\left(r_{1}\right) \rho\left(r_{2}\right) \rho\left(r_{3}\right) d^{3} \mathbf{r} d^{3} \mathbf{r}_{1} d^{3} \mathbf{r}_{2} d^{3} \mathbf{r}_{3} .
\end{aligned}
$$

As follows from the relations (3) and (5) the first term of the rhs is

$$
\mathcal{E}_{s t}^{(1)}=\sum_{\lambda} n_{\lambda} \varepsilon_{\lambda}
$$

But as seen from Eq.(17) and the sum rule

$$
\varepsilon_{\lambda}=\sum_{j}^{(A-1)} E_{j} s_{j}^{(\lambda)}+\sum_{k}^{(A+1)} E_{k} s_{k}^{(\lambda)}
$$

for the doorway state energies, see Eq.(14c) of Ref.[1], the quantity $\mathcal{E}_{s t}^{(1)}$ contains unphysical contributions from the energies of the $(A+1)$ nuclear states. To eliminate them let us divide $\mathcal{E}_{s t}^{(1)}$ into the two parts

$$
\mathcal{E}_{s t}^{(1)}=\mathcal{E}_{s t}^{<}+\mathcal{E}_{s t}^{>}, \quad \mathcal{E}_{s t}^{<}=\sum_{\lambda \leq F} n_{\lambda} \varepsilon_{\lambda}, \quad \mathcal{E}_{s t}^{>}=\sum_{\lambda>F} n_{\lambda} \varepsilon_{\lambda}
$$

including summations over the states with $\varepsilon_{\lambda} \leq \varepsilon_{F}$ and $\varepsilon_{\lambda}>\varepsilon_{F}$ respectively. The doorway states entering $\mathcal{E}_{\text {st }}^{<}$are mainly distributed over the states of the $(A-1)$ nucleus the first term 
of Eq.(22) thus giving the dominant contribution to the $\varepsilon_{\lambda}$ values in this case. The small unphysical contribution from the second term of $(22)$ arises from the states of the $(A+1)$ nucleus with the same quantum numbers as those of the low-lying states of the $(A-1)$ nucleus. Such states lie either in the continuum or near its border. Therefore the reasonable estimate for their energies is $E_{k} \approx 0$ the unphysical contribution thus being negligible for this case.

The situation is opposite for $\mathcal{E}_{s t}^{>}$because the doorway states with $\varepsilon_{\lambda}>\varepsilon_{F}$ are mainly distributed over the states of the $(A+1)$ nucleus, the dominant contribution to the $\varepsilon_{\lambda}$ values thus being unphysical from the viewpoint of the binding energy. In such conditions it is reasonable to use a more appropriate relation for $\mathcal{E}_{s t}^{>}$,

$$
\widetilde{\mathcal{E}}_{s t}^{>}=\sum_{\lambda>F} \sum_{j}^{(A-1)} E_{j} s_{j}^{(\lambda)}
$$

So the $(A-1)$ nuclear states with the same quantum numbers as those of the low-lying states of the $(A+1)$ nucleus contribute in this case. There are no such states among the low-lying ones of the $(A-1)$ nucleus, and therefore

$$
E_{j}<E_{F}=\mathcal{E}_{0}(A)-\mathcal{E}_{g}(A-1)
$$

see Ref. [1] for the details. Nevertheless it is reasonable to use the estimate

$$
E_{j}=E_{F}
$$

providing the least absolute value for the $\widetilde{\mathcal{E}}_{\text {st }}^{>}$quantity. In this way we get

$$
\widetilde{\mathcal{E}}_{s t}^{>}=E_{F} \sum_{\lambda>F} \sum_{j}^{(A-1)} s_{j}^{(\lambda)}=E_{F} \sum_{\lambda>F} n_{\lambda}=E_{F} \widetilde{N}_{\text {out }}
$$

Accounting for the fact that the many-particle forces are introduced as the contact ones in the model-independent approach of Ref.[1], we finally get (see Ref. [1] for the notations)

$$
\begin{aligned}
\mathcal{E}_{\text {st }}= & \sum_{i=n}^{p}\left(\sum_{\lambda \leq F} n_{\lambda} \varepsilon_{\lambda}+\widetilde{N}_{\text {out }} E_{F}\right)_{i}- \\
- & \frac{1}{2} \int\left(S(r) \rho_{s}(r)+V_{\omega}(r) \rho(r)+S^{-}(r) \rho_{s}^{-}(r)+V_{\rho}(r) \widetilde{\rho}^{-}(r)+C(r) \rho_{c h}(r)\right) d^{3} \mathbf{r}- \\
- & \int\left(\frac{2}{3} a_{3} \rho^{3}(r)+\frac{3}{4} a_{4} \rho^{4}(r)+\frac{1}{2}\left[a_{3}^{-} \rho(r)+a_{4}^{-} \rho^{2}(r)\right]\left(\rho^{-}(r)\right)^{2}\right] d^{3} \mathbf{r} .
\end{aligned}
$$

We used the following ansatz for the occupation numbers

$$
n_{\lambda}=\frac{1}{2}\left[1-\frac{\varepsilon_{\lambda}-\mu}{\sqrt{\left(\varepsilon_{\lambda}-\mu\right)^{2}+C^{2}}}\right], \quad \sum_{\lambda} n_{\lambda}=N, \quad \sum_{\lambda>F} n_{\lambda}=\widetilde{N}_{\text {out }}
$$

putting $\widetilde{N}_{\text {out }}=N_{\text {out }}$, see Sect.2. This is incorrect because the sum $\sum_{\lambda>F} n_{\lambda}$ is different from $N_{\text {out }}$, Eq.(9). Indeed, both diagonal and nondiagonal elements of the density matrix, Eq.(4), 
Table 2: The static and observed binding energies in $\mathrm{MeV}$ together with the dominant contributions to $\mathcal{E}_{s t}$. Those from the Coulomb and the isovector terms are not shown, but they are included into the $\mathcal{E}_{s t}$ value. The notations correspond to the terms of Eq.(28).

\begin{tabular}{rrrrrrrr}
\hline \hline & $\mathcal{E}_{s t}^{(1)}$ & $-\frac{1}{2} S$ & $-\frac{1}{2} V_{\omega}$ & $-\frac{2}{3} a_{3}$ & $-\frac{3}{4} a_{4}$ & $\mathcal{E}_{s t}$ & $\mathcal{E}_{b}$ \\
\hline${ }^{16} \mathrm{O}$ & -345.6 & +1528.4 & -1224.0 & -451.2 & +461.8 & -47.0 & -127.6 \\
${ }^{40} \mathrm{Ca}$ & -991.7 & +4456.0 & -3570.8 & -1346.0 & +1411.3 & -120.7 & -342.0 \\
${ }^{90} \mathrm{Zr}$ & -2251.6 & +10699.0 & -8565.6 & -3236.5 & +3386.0 & -234.2 & -783.9 \\
${ }^{208} \mathrm{~Pb}$ & -4998.2 & +26463.4 & -21255.6 & -8190.9 & +8694.0 & -233.3 & -1636.5 \\
\hline \hline
\end{tabular}

contribute to the $N_{\text {out }}$ values. This shortcoming will be corrected in future by calculating the $n_{\lambda}$ and $\rho_{\lambda \nu}$ values, see the discussion in Sect.2 (of course, the ansatz (29) will become unnecessary).

The results of the calculations are shown in Table 2. As seen from the table the static energy is the sum of a number of contributions with different sign, the dominant ones greatly exceeding the total static energy as well as the observed binding one. This is the source of the ambiguities because all disregarded effects may be of importance in such a situation.

One of such effects is the finite range of the many-particle forces. The possible way of the estimation is illustrated for the three-particle forces. As seen from Eq.(10) the original contribution is

$$
\begin{aligned}
\mathcal{E}_{3} & =\frac{1}{3} \iiint f_{3}\left(\left|\mathbf{r}-\mathbf{r}_{1}\right|,\left|\mathbf{r}-\mathbf{r}_{2}\right|\right) \rho(r) \rho\left(r_{1}\right) \rho\left(r_{2}\right) d^{3} \mathbf{r} d^{3} \mathbf{r}_{1} d^{3} \mathbf{r}_{2}= \\
& =\frac{1}{3} \iiint \rho(r) f_{3}(\xi, \eta) \rho(|\mathbf{r}+\boldsymbol{\xi}|) \rho(|\mathbf{r}+\boldsymbol{\eta}|) d^{3} \mathbf{r} d^{3} \boldsymbol{\xi} d^{3} \boldsymbol{\eta} \cong \\
& \cong \frac{1}{3} \iiint \rho(r) f_{3}(\xi, \eta)\left[\rho(r)+\boldsymbol{\xi} \boldsymbol{\nabla} \rho(r)+\frac{1}{2} \xi_{1} \xi_{k} \partial_{i} \partial_{k} \rho(r)+\cdots\right] \times \\
& \times\left[\rho(r)+\boldsymbol{\eta} \boldsymbol{\nabla} \rho(r)+\frac{1}{2} \eta_{j} \eta_{\ell} \partial_{j} \partial_{\ell} \rho(r)+\cdots\right] d^{3} \mathbf{r} d^{3} \boldsymbol{\xi} d^{3} \boldsymbol{\eta}= \\
& =\frac{1}{3} \iiint \rho(r) f_{3}(\xi, \eta)\left[\rho(r)+\frac{1}{6} \xi^{2} \Delta \rho(r)+\cdots\right]\left[\rho(r)+\frac{1}{6} \eta^{2} \Delta \rho(r)+\cdots\right] d^{3} \mathbf{r} d^{3} \boldsymbol{\xi} d^{3} \boldsymbol{\eta}= \\
& =\frac{1}{3} a_{3} \int \rho(r)\left[\rho(r)+r_{0}^{2} \Delta \rho(r)+\cdots\right]^{2} d^{3} \mathbf{r} \cong \frac{1}{3} a_{3} \int \rho^{3}(r) d^{3} \mathbf{r}+\frac{2}{3} a_{3} \int \rho^{2}(r) \Delta \rho(r) d^{3} \mathbf{r},
\end{aligned}
$$

where (see Eq.(37) of Ref.[1])

$$
a_{3}=\iint f_{3}(\xi, \eta) d^{3} \boldsymbol{\xi} d^{3} \boldsymbol{\eta}, \quad a_{3} r_{0}^{2}=\frac{1}{6} \iint \xi^{2} f_{3}(\xi, \eta) d^{3} \boldsymbol{\xi} d^{3} \boldsymbol{\eta}=\frac{1}{6} \iint \eta^{2} f_{3}(\xi, \eta) d^{3} \boldsymbol{\xi} d^{3} \boldsymbol{\eta} .
$$

In this way we get

$$
\begin{aligned}
& \delta_{r_{0}^{2}} \mathcal{E}_{s t}=r_{0}^{2} \int\left(\frac{2}{3} a_{3} \rho^{2}(r)+\frac{3}{4} a_{4} \rho^{3}(r)\right) \Delta \rho(r) d^{3} \mathbf{r}=-D r_{0}^{2} \\
& D=\int\left(\frac{4}{3} a_{3} \rho(r)+\frac{9}{4} a_{4} \rho^{2}(r)\right)\left(\rho^{\prime}(r)\right)^{2} d^{3} \mathbf{r} .
\end{aligned}
$$

The calculated $D$ values are shown in Table 3 . The reasonable value of the range parameter is the $\omega$ meson Compton wavelength, i.e. a typical scale of the strong interaction. So $r_{0}^{2}=$ $m_{\omega}^{-2}=0.1 \mathrm{fm}^{2}$, the effect thus being negligibly small. 
Table 3: The many-particle finite range effect

\begin{tabular}{lcccc}
\hline \hline & ${ }^{16} \mathrm{O}$ & ${ }^{40} \mathrm{Ca}$ & ${ }^{90} \mathrm{Zr}$ & ${ }^{208} \mathrm{~Pb}$ \\
$D, \mathrm{MeV} \cdot \mathrm{fm}^{-2}$ & -12.12 & -3.29 & 22.02 & 34.08 \\
& & & & \\
\hline \hline
\end{tabular}

Table 4: The contributions to the static field

\begin{tabular}{cccc}
\hline \hline & $U_{2}$ & $U_{3}$ & $U_{4}$ \\
& & & \\
Bonn B & -83 & +96.5 & -104 \\
OSBEP & -82 & +97 & -107 \\
\hline \hline
\end{tabular}

The second source of the possible ambiguity is seen from the results of Ref. [1] for the contributions of two-particle, three-particle and four-particle forces to the nuclear static field which are shown in Table 4, see Eqs. (44) and (45) of Ref. [1].

As follows from the table the convergence of this sequence is not seen. This observation suggests the possible existence of the contributions from higher many-particle forces, and therefore the whole sequence should be summed up. But this can be done only within some reasonable model for the many-particle forces including all higher-order ones as well as the finite range and the mechanism for the saturation of nuclear density. The corresponding investigation is in progress.

\section{References}

[1] B.L. Birbrair and V.I. Ryazanov, nucl-th/9907058.

[2] R. Machleidt, nucl-th/9911059.

[3] F. Klingl and W. Weise, nucl-th/9802211.

[4] G.E. Brown et al., nucl-th/9806026.

[5] S. Leopold and U. Mosel, nucl-th/9805024.

[6] A.M. Rakhimov et al., nucl-th/9806062.

[7] J.L. Friar, nucl-th/9911075.

[8] J.D. Walecka, Ann.of Phys. (NY) 83 (1974) 491.

[9] A.B. Migdal, "Theory of finite Fermi-systems and properties of atomic nuclei", M, 1983. 
[10] L.L Frankfurt and M.I. Strikman, Phys.Rep. 76 (1981) 215.

[11] L.L. Frankfurt, M.I. Strikman, D.B. Day and M. Sargsyan, Phys.Rev. C48 (1993) 2451.

[12] V.A. Khodel and V.R. Shaginyan, Particles and Nuclei (Dubna) 22 (1991) 436. 